and International Conference Art, Illustration and Visual Culture in Infant and Primary Education

\title{
Drawing unusual objects after making their acquaintance by touch
}

Sérgio Carvalho10orespostas@gmail.com Liliana Couto lilianalvescouto@hotmail.com Universidade de Aveiro, Portugal

Reference

Carvalho, Sérgio; Couto, Liliana; (2012) "Drawing unusual objects after making their acquaintance by touch", p. 251-255 . In: Barbosa, Helena; Quental, Joana [Eds]. Proceedings of the 2nd International Conference of Art, Illustration and Visual Culture in Infant and Primary Education. São Paulo: Blucher, 2015. ISSN 2318-695X, ISBN: 978-989-98185-0-7 DOI 10.5151/edupro-aivcipe-48

The drawing is thought of as a visual manifestation of a sensation and a visual indicator of the intellectual development of man; there is a correlation between the ability to design and visualization or composition skills. The education system is based on a process of artistic creation based mainly on the sense of sight. This raises a question: how do children, endowed with sense of vision, draw unusual objects with reference only to the memory of its touch? This research takes as a sample a group of adolescent children, and submits it to a design experience based only on memories of touch sensations. We tried to understand what influence the memories and prior knowledge of these subjects had on the projections of objects. In a global context in which the privileged senses are vision and hearing, we have the goal to explore the sense of touch as a source of cognitive development.

\section{Theoretical background}

Not every human cognitive experience demands the use of all 5 senses. In fact some senses are obsolete in some situations, and most of the time a person must select specific stimulus inputs in order to be able to process those (Styles, 2005:6). Most of the time the knowledge building takes place as information from 2 or more senses is gathered simultaneously in our memory.

Before entering the official educational system children use all their senses to learn and interact with the world within their grasp. As they get entangled in the school's criteria they are inclined to favour vision and hearing as the dominant sensory tools to incorporate the curriculum as touch is overlooked.

In the field of illustration vision has the main function of translating sensory data provided by the shapes of objects. And yet, sighted people can quickly identify objects in a matter of seconds merely by feeling their tactile features with their hands (Lederman \& Metzger, 1985).

Images come before words when the issue is learning (Rollins, 2005:209). This works for reconnaissance as well as graphical representation of memorized visual shapes. Although almost every person can recognize a shape, most of them cannot draw that shape with detail despite being very familiar with it. This is because "[...] recognition memory [...] [is] a more basic cognitive skill" (Rowland \& Schweigert, 2000:6). The sense of touch performs a complementary source of information that establishes the connection between what we see and what we feel through the skin, meaning objects can be thoroughly explored through touch (Kurze, 1997:4). In the next sub section we will look deeper into this process. 
2nd International Conference Art, Illustration and Visual Culture in Infant and Primary Education $2^{\circ}$ Congreso Internacional

Arte, Ilustración y Cultura Visual en Educación Infantil y Primaria
Congresso Internacional

de Arte, llustracão e Cultura Visual

na Educação Infantil e Primária

\subsection{Haptic experience and drawing}

\subsection{Methodology}

Figure1, 2 and 3 . Three of the 14 objects used in the investigation, manufactured in April 2012. Source: personal.
An image is a quicker source of information input. If compared to text one can say the former is a parallel input while the latter is a serial input. Hence it can be stated that "externalization seems to be a process which can be used to restructure uninterpretable input. In the case of line drawings, it restructures the serial input into parallel input"' (Wijntjes et al., 2008:262). Drawing is a simulation of a reality we cannot replicate with every detail. It's a way to transmit, through a symbolic system, an idea of something that's inaccessible to the eye at that moment (Rowland \& Schweigert, 2000:3); it's one of the primordial ways of expressing emotions and personality (Rollins, 2005:204) while one still has no verbal skills to do so. Around the age of 8-10, one begins to interpret the visual input as a narrowing realistic picture. Afterwards the sense of depth and the correspondent graphical depiction arise.

The word haptic is an assemblage of two sub systems: the tactile and the kinaesthetic perception. The first, consisting on the skin, is called sensory and it deals with textures, and temperatures; the second, consisting on muscles and joints, is called the manipulatory, and it deals with three-dimensional shapes (Lederman \& Klatzky, 1987).

The haptic experience, in a situation where the subject is deprived of the sense of vision, calls upon finger movements for a parallel perception mode. The identification of an objects shape would be compromised if it was made by serial perception (e. g. a single finger). This means parallel reconnaissance of object lines is undoubtedly more reliable, not only through visual but also haptic processes (Wijntjes et al., 2008:256).

Viewing only requires a fraction of a second to register a shape, while touching needs finger motion. The interesting thing is that "evidence suggests that haptic processing recruits visual areas [in the brain] in order to construct a representation of an object" (Amedi et al., 2008:2), meaning there is a link between vision and touch. If someone who had just haptically explored an object as prompted to draw it, one may ask: what kind of representation would the outcome be? This study was conducted bearing this very question in mind.

The sample group was composed of 24 individuals attending the 7 th grade with an average age of 13 years and 4 months.

14 objects were hand-made to fulfill the material needs for this investigation. These objects, made of plaster, consisted of simple geometric shapes with holes or as assemblage of two basic solid shapes (see Figure 1, 2 and 3).
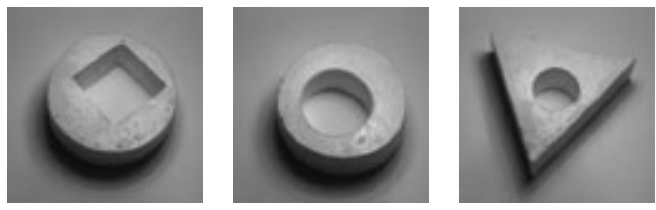

They were placed inside card board boxes and handed at random to the subjects. Then these were asked to draw 3 pictures of their object. The first was meant to be based of the short term tactile memory they obtained from touching the object. The second was made while simultaneously grabbing the object. The last one was made with the object in sight of the subject. After this procedure the 3 drawings of each pupil were compared and rated according to the following criteria:

- Display (or not) of the objects general morphology;

- Display (or not) of the proportionality of the parts and the whole of the object;

- Display of 3D graphical hint (or 2D);

- Presence (or absence) of shading on the object's surface rendering;

The results were expected to provide us with an argument about the effectiveness of object haptic interpretation through drawing. 
and International Conference Art, Illustration and Visual Culture in Infant and Primary Education
Congressolnternacional

de Arte, llustração e Cultura Visual

na Educação Infantil e Primária

\subsection{Procedure}

The experiment took place on two separate days with two different groups. On both occasions the experiment took 90 minutes to perform. The first day had 10 subjects and the second day had 14 subjects. The week before this study was undertaken the pupils were taught to use shading effects to give the impression of volume to their drawings. Pupils used only graphite pencils for the 3 drawings of the experiment.

The solid geometric shapes were placed inside cardboard boxes built for the purpose of this experiment. The boxes were named after a letter and randomly distributed to the pupils. The subjects were then instructed to open the box without looking inside it. The following step was for them to place their left hand inside the box and feel the shape which was inside of it.

The first part of the experiment consisted of two distinct moments. First the subjects had to feel the shape with their hands without ever looking at it. Second, the subjects closed the boxes and for 20 minutes drew the objects based on the memory they had collected from their first haptic experience.

The second part of the experiment took place immediately after the first. Now the subjects had to draw the same shape and touch it simultaneously. Hence, they placed their left hand inside the box, without peeking inside, and drew it with their right hand. This task also took around 20 minutes to complete.

The third part of the experiment was performed right afterwards. This time the subjects had to draw the object by looking at it. For this drawing they had no need to touch the object but to place it outside the box in a position favourable to the task. 20 minutes was the time for completion.

As it was mentioned before, this very same three part procedure was adopted on another occasion, two days later, with another group of 14 pupils. Thus we ended up testing a sample group of 24 individuals and collected 72 drawings to classify based on the 4 items listed in the previous subsection.

On the first phase all drawings displayed the general object shape although this percentage decreased slightly on the two following phases (see table 1). The observance of proportionality reached its top score on the second phase. Even though the difference is gentle, one would expect the third phase to reflect a much more accurate display of the object's proportionality.

The illusion of three dimensions was a dominant feature overall. Despite some ambiguous drawings which could not be considered as displaying a third dimension in the two first phases, the final one boosts the three-dimensional sensation to a $100 \%$.

As for the aspect of shading features, one can only observe steadiness. All three phases presented the same percentage of $96 \%$ existence of shades on the objects surface. What wasn't so stable was their consistency with the shape. At first most subjects were incapable of representing the shadows according to the shape. This performance slightly improved on the second phase but came down on the final phase. This is unexpected since all subjects could see their object's true visual appearance. A discussion about these results is called upon.

\begin{tabular}{llccc}
\hline Drawing properties & & $\mathbf{1}^{\text {st }}$ phase & $\mathbf{2}^{\text {nd }}$ phase & $3^{\text {rd }}$ phase \\
\hline \multirow{2}{*}{ Display of the objects general morphology } & Yes & $100 \%$ & $87 \%$ & $92 \%$ \\
\cline { 2 - 5 } & No & $0 \%$ & $12 \%$ & $8 \%$ \\
\hline $\begin{array}{l}\text { Display of the proportionality of the parts and } \\
\text { the whole of the object }\end{array}$ & Yes & $29 \%$ & $12 \%$ & $21 \%$ \\
\cline { 2 - 5 } No & $71 \%$ & $87 \%$ & $79 \%$ \\
\hline \multirow{2}{*}{$\begin{array}{llcl}\text { Display of 3D graphical hint } \\
\text { Presence of shading on the object's surface } \\
\text { rendering }\end{array}$} & Yes & $92 \%$ & $83 \%$ & $100 \%$ \\
\cline { 2 - 5 } & Yes & $96 \%$ & $96 \%$ & $96 \%$ \\
\cline { 2 - 5 } & No & $4 \%$ & $4 \%$ & $4 \%$ \\
\hline
\end{tabular}


$2^{\text {nd }}$ International Conference Art, Illustration and Visual Culture in Infant and Primary Education

\subsection{Discussion}

Figures 4, 5, 6, 7, 8 and 9. Comparison between phase 2 and 3. Two on the left made by subject 13_F, two on the middle made by subject 09_C, two on the right made by subject 11_C. Source: personal.

\section{Conclusions}

As we analyse these results it becomes evident that the cognitive development plays a major role in the drawing performance. This is to say pictures drawn by infants "could become a means of diagnosing intellectual development" (Kitahara \& Matsuishi, n/d:11). The skills and drawing strategies are intimately related to the memory of familiar objects. We found that subjects who had a minor academic performance were those with the less capability for portraying the object at hand.

In the comparison between the first two phases and the third one we acknowledged some relevant shape features interpretation differences:

- When a shape had a hole across, many subjects tended to believe that the hole's location was at the centre of the shape's contour. As they drew it the third time they carefully placed the hole in its correct position (figures 4 and 5). We assume the first two are the result of a mental image simplification.

- In some subject's depiction the first two drawings are a flat or divergent synthesis of the object. As for the third drawing, it features an idea of depth through perspective and shading (figures 6 and 7).

- Another common approach is the maintenance, on the 3 drawings, of the graphic configuration overlooking the basic rules of perspective even when the subjects are looking straight at the object (figures 8 and 9).
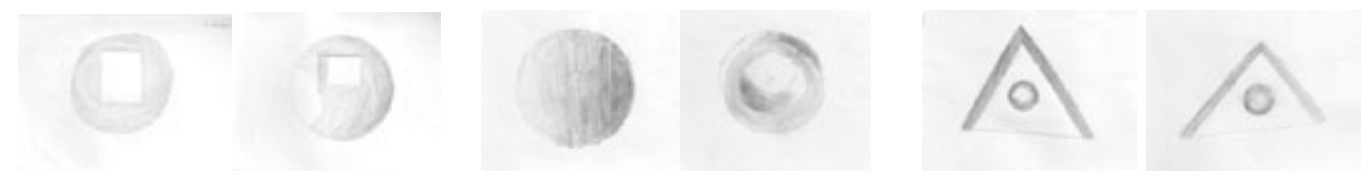

Generally speaking the haptic experience functions very well for object recognition. Subjects demonstrated a swift ability to recognise basic geometric shapes by merely touching them. But the major tendency, especially with younger subjects, was the taking up of a cognitive synopsis of shapes. The holes on some objects were deliberately placed off centre and oblique to the outer borders, but most subjects did not depict those details while drawing them. They insisted on the simplification of such asymmetries. We believe the reason for this is the existence of visual prototypes in the subjects geometric shape mental processing. Their prevailing line of thinking is one that fits shapes into "visual wholes", without attending to small nuances. Also, the inverted perspectives of objects revealed a still naïve assortment of mental geometric shape imagery.

As for shading, the dominant graphic representation technique was also analytic instead of synthetic. For different parts of the objects we often found different shading renderings, as if each part of it was lit by a different light source. Oddly, looking at the object didn't inspire them to perpetrate more accurate shadings. It's as if they only believed in what they already believed to be correct - the prototypes - and not in what they were looking at.

In summary, this experiment showed that it's easy for an early teenage child to haptically identify a shape's morphology, although it's not so easy for him/her to draw it in a conventional realistic way. But pupil's spatial abilities can gain a significant increase if they are prompted to methodically "look" at objects, not so much with their eyes, but with their fingers. 


\section{Bibliographical references}

Amedi, A., et al.: Neural and behavioral correlates of drawing in an early blind painter: a case study. [on line] 2008. [accessed on April 9, 2012]. Available at <http://www.sciencedirect.com/ science/article/pii/Sooo6899308018398>

Kitahara, R. \& Matsuishi, T.: Research on Children's Drawings. [on line] n/d [accessed on Fedruary 10, 2012]. Available at < http://www.medico-pedagogy.org/childrenpicturesummaryJ_ Ewp.pdf>

Kurze, M.: Rendering Drawings for Interactive Haptic Perception. [on line] February 1997. [accessed on April 2, 2012]. Available at <ftp://ftp.cs.york.ac.uk/irs/iss/iss_wp11.rtf>.

Lederman, S. \& Metzger, V. (1985). "Identifying objects by touch: an 'expert system'”. Perception \& Psychophysics, Vol. 37, n. 4, pp. 299-302.

Lederman, S. J. \& Klatzky, R. L. (1987). "Hand movements: a window into haptic object recognition". Cognitive psychology, vol. 19, pp. 342-368.

Lederman, S. J., Klatzky, R. L. \& Barber, P.O. (1985). "Spatial and movement-based heuristics for encoding pattern information through touch". Journal of Experimental Psychology: General, Vol. 114, n. 1, pp.33-49.

Rollins, J. A. (2005). "Tell Me About It: Drawing as a Communication Tool for Children With Câncer". Journal of Pediatric Oncology Nursing, Vol. 22, n. 4 pp. 203-221.

Rowland, C. \& Schweigert, P.: Tangible Symbol Systems. Making the Right to Communicate a Reality for Individuals with Severe Disabilities [on line]. 2000. [acessed on April 9, 2012]. Available at <http://www.osepideasthatwork.org/toolkit/pdf/TangibleSymbol\%2oSystems.pdf> Styles, E. A. (2005). Attention, Perception and Memory - An Integrated Introduction. New York: Psychology Press.

Wijntjes, M.W.A. et al. (2008). "Look what I have felt: Unidentified haptic line drawings are identified after sketching”. Acta psychologica, n. 128, pp. 255-263. 